TRANSACTIONS OF THE

AMERICAN MATHEMATICAL SOCIETY

Volume 358, Number 12, December 2006, Pages 5425-5439

S $0002-9947(06) 03867-0$

Article electronically published on April 11, 2006

\title{
THE BRAID INDEX IS NOT ADDITIVE FOR THE CONNECTED SUM OF 2-KNOTS
}

\author{
SEIICHI KAMADA, SHIN SATOH, AND MANABU TAKABAYASHI
}

\begin{abstract}
Any 2-dimensional knot $K$ can be presented in a braid form, and its braid index, $\operatorname{Braid}(K)$, is defined. For the connected sum $K_{1} \# K_{2}$ of 2-knots $K_{1}$ and $K_{2}$, it is easily seen that $\operatorname{Braid}\left(K_{1} \# K_{2}\right) \leq \mathrm{B}\left(K_{1}\right)+\mathrm{B}\left(K_{2}\right)-1$ holds. Birman and Menasco proved that the braid index (minus one) is additive for the connected sum of 1-dimensional knots; the equality holds for 1-knots. We prove that the equality does not hold for 2 -knots unless $K_{1}$ or $K_{2}$ is a trivial 2-knot. We also prove that the 2-knot obtained from a granny knot by Artin's spinning is of braid index 4 , and there are infinitely many 2 -knots of braid index 4 .
\end{abstract}

\section{INTRODUCTION}

By the Alexander theorem [1, any 1-dimensional knot $k$ can be presented in a braid form. The braid index of $k$ is the minimum number among the degrees of all closed braids that are equivalent to $k$, which is denoted by $\operatorname{Braid}(k)$. When 1-knots $k_{1}$ and $k_{2}$ are presented by an $m_{1}$-braid $b_{1}$ and an $m_{2}$-braid $b_{2}$ respectively, one can construct an $\left(m_{1}+m_{2}-1\right)$-braid $b_{1} \# b_{2}$ which presents the connected sum $k_{1} \# k_{2}$. Thus there is an obvious inequality

$$
\operatorname{Braid}\left(k_{1} \# k_{2}\right) \leq \operatorname{Braid}\left(k_{1}\right)+\operatorname{Braid}\left(k_{2}\right)-1 .
$$

Actually, J. S. Birman and W. Menasco [4] proved that the braid index (minus one) is additive under the connected sum.

Theorem 1 (Birman-Menasco [4). For the connected sum $k_{1} \# k_{2}$ of 1 -knots $k_{1}$ and $k_{2}$,

$$
\operatorname{Braid}\left(k_{1} \# k_{2}\right)=\operatorname{Braid}\left(k_{1}\right)+\operatorname{Braid}\left(k_{2}\right)-1 .
$$

In this paper we consider an analogous problem for 2-knots. The notion of a 2 dimensional braid was introduced by O. Ya. Viro 29, and it is proved in 12 that any 2-knot (or any closed oriented 2-submanifold of $\mathbb{R}^{4}$ ) can be presented in a braid form. A similar notion, called a braided surface, was studied by L. Rudolph 25, 26, 27. J. S. Carter and M. Saito have been studying the braid presentations of 2-knots, [5, 6, 7, 8. Refer to [10, 11, 12, 13, 16, 18, 19] for the first author's research in this field. Another kind of braid presentation of a 2-knot was studied by F. GonzálezAcuña [9]. 2-dimensional braids are also related to the braid monodromies of stable branch curves in complex geometry [22, 23].

Received by the editors July 15, 2003 and, in revised form, October 1, 2004.

2000 Mathematics Subject Classification. Primary 57Q45.

Key words and phrases. Braid index, 2-knots, surface-knots, 2-dimensional braids, braided surfaces, charts.

(C)2006 American Mathematical Society Reverts to public domain 28 years from publication 5425 
The braid index, which is denoted by $\operatorname{Braid}(K)$, of a 2 -knot $K$ is defined to be the minimum number among the degrees of all simple closed 2-dimensional braids that are equivalent to $K$. The braid index is 1 if and only if $K$ is a trivial 2-knot. There exist no 2-knots of braid index 2 (cf. [10]). It is proved in [10] that if the braid index of a 2 -knot $K$ is 3 , then $K$ is a ribbon 2 -knot, that is, it can be obtained from some mutually disjoint trivial 2 -spheres in $\mathbb{R}^{4}$ by surgery along some 1-handles; cf. 20. The converse is not true; for example, the 2-knot obtained from a figure eight knot by Artin's spinning construction [2] is ribbon, and the braid index is not 3, 16.

For a 2 -dimensional $m_{1}$-braid $S_{1}$ and a 2 -dimensional $m_{2}$-braid $S_{2}$, we can construct a 2-dimensional $\left(m_{1}+m_{2}-1\right)$-braid $S_{1} \# S_{2}$ such that the closure in $\mathbb{R}^{4}$ is equivalent to the connected sum of the closures of $S_{1}$ and $S_{2}$. The following is just an analogy of the inequality (11).

Lemma 2 ([17]). For 2 -knots $K_{1}$ and $K_{2}$,

$$
\operatorname{Braid}\left(K_{1} \# K_{2}\right) \leq \operatorname{Braid}\left(K_{1}\right)+\operatorname{Braid}\left(K_{2}\right)-1,
$$

where $K_{1} \# K_{2}$ is the connected sum of $K_{1}$ and $K_{2}$.

It is obvious that

$$
\operatorname{Braid}\left(K_{1} \# K_{2}\right)=\operatorname{Braid}\left(K_{1}\right)+\operatorname{Braid}\left(K_{2}\right)-1,
$$

provided that $K_{1}$ or $K_{2}$ is a trivial 2 -knot. Surprisingly, this is the only case where the equality of (2) holds for the connected sum of 2-knots. The following theorem is our main result.

Theorem 3. If neither $K_{1}$ nor $K_{2}$ is a trivial 2-knot, then

$$
\operatorname{Braid}\left(K_{1} \# K_{2}\right)<\operatorname{Braid}\left(K_{1}\right)+\operatorname{Braid}\left(K_{2}\right)-1
$$

There exist infinitely many ribbon 2-knots of braid index 3 (cf. Lemma 15] or [16]). Using Theorem 3, we prove the following.

Corollary 4. There exist an infinite series of ribbon 2-knots of braid index 4 which includes the 2-knot obtained from a granny knot by Artin's spinning.

This paper is organized as follows. In Sections 2 and 3, we review the notions of a 2-dimensional braid and the chart presentation. In Sections 4 and 5, we prove Theorem 3 by using the chart presentation. A proof of Corollary 4 is given in Section 6 .

\section{Simple 2-Dimensional BRAids}

Let $D_{1}$ and $D_{2}$ be 2-disks and $X_{m}$ a fixed set of $m$ distinct interior points of $D_{1}$, where $m$ is a positive integer. Let $\pi: D_{1} \times D_{2} \rightarrow D_{2}$ be the projection map. A 2-dimensional $m$-braid (or a 2-dimensional braid of degree $m$ ) is a compact oriented surface $S$ properly embedded in $D_{1} \times D_{2}$ such that

(i) the restriction map $\left.\pi\right|_{S}: S \rightarrow D_{2}$ of the projection $\pi$ to $S$ is an $m$-fold branched covering map of $D_{2}$, and

(ii) the boundary $\partial S$ is the trivial closed $m$-braid $X_{m} \times \partial D_{2}$ in the solid torus $D_{1} \times \partial D_{2}$. 
Moreover, if an additional condition that

(iii) the branched covering $\left.\pi\right|_{S}: S \rightarrow D_{2}$ is simple, that is, $\#\left(S \cap \pi^{-1}(x)\right)=$ $m-1$ or $m$ for each $x \in D_{2}$,

is satisfied, then the 2-dimensional $m$-braid $S$ is said to be simple.

Two simple 2-dimensional braids $S$ and $S^{\prime}$ are equivalent if they are ambiently isotopic by an ambient isotopy $\left\{h_{t}\right\}_{t \in[0,1]}$ of $D_{1} \times D_{2}$ such that for each $t \in[0,1]$, $h_{t}(S)$ is a simple 2-dimensional braid. This definition is different from that in 10, however these definitions are equivalent [14. Moreover, it is proved in 14] that simple 2-dimensional $m$-braids $S$ and $S^{\prime}$ are equivalent if and only if there exists a one-parameter family $\left\{S_{t}\right\}_{t \in[0,1]}$ of simple 2-dimensional $m$-braids with $S_{0}=S$ and $S_{1}=S^{\prime}$. We usually regard equivalent 2-dimensional braids as the same.

Divide the 2-disk $D_{2}$ into two 2-disks $D_{2}^{(1)}$ and $D_{2}^{(2)}$ by a properly embedded arc in $D_{2}$. For simple 2-dimensional $m$-braids $S_{1}$ and $S_{2}$, we define the product $S_{1} \cdot S_{2}$ to be the simple 2-dimensional $m$-braid such that the restriction to $D_{1} \times D_{2}^{(i)}$ is a copy of $S_{i}$ for each $i \in\{1,2\}$. This product is uniquely determined by $S_{1}$ and $S_{2}$ up to equivalence.

Let $S$ be a simple 2-dimensional $m$-braid. Identify $D_{2}$ with the product $I_{1} \times I_{2}$ of the unit intervals $I_{1}$ and $I_{2}$. For each $t \in I_{2}=[0,1]$, we denote the cylinder $D_{1} \times I_{1} \times\{t\} \subset D_{1} \times D_{2}$ by $(D \times I)_{t}$ and put $b_{t}=S \cap(D \times I)_{t}$. Then $b_{t}$ is a 1-dimensional $m$-braid in $(D \times I)_{t}$ for all $t$ but a finite number of exceptional values. For each exceptional value $t$, the singular braid $b_{t}$ has double points in its strands; see Figure 1. We call the one-parameter family $\left\{b_{t}\right\}_{t \in[0,1]}$ a motion picture of $S$. Note that both $b_{0}$ and $b_{1}$ are trivial $m$-braids, since $\partial S=X_{m} \times \partial D_{2} \subset D_{1} \times D_{2}$.

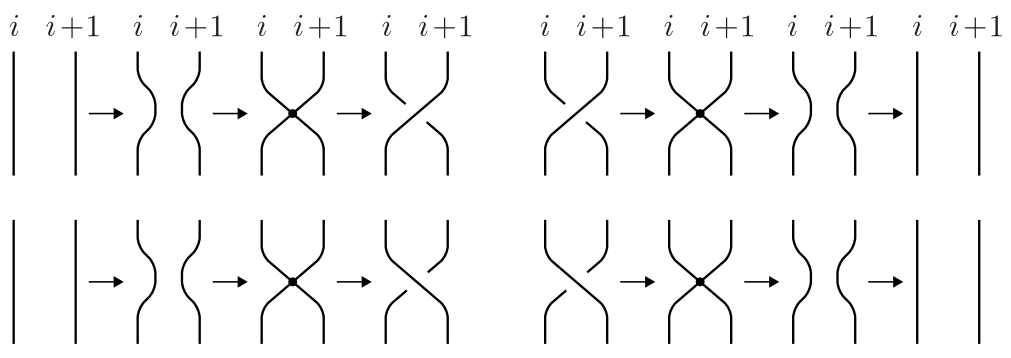

FIGURE 1.

When $\left\{b_{t}^{(1)}\right\}_{t \in[0,1]}$ and $\left\{b_{t}^{(2)}\right\}_{t \in[0,1]}$ are motion pictures of $S_{1}$ and $S_{2}$ respectively, we have a motion picture of the product $S_{1} \cdot S_{2}$ of $S_{1}$ and $S_{2}$ by taking the usual product $b_{t}^{(1)} \cdot b_{t}^{(2)}$ as braids for each $t \in[0,1]$.

Embed the 4 -disk $D_{1} \times D_{2}$ in $\mathbb{R}^{4}$. Let $S$ be a 2 -dimensional $m$-braid. The boundary of $S$ is a trivial link in the 3 -sphere $\partial\left(D_{1} \times D_{2}\right)$. Attaching $m$ sheets of 2-disks along the boundary of $S$ in $\mathbb{R}^{4} \backslash \operatorname{int}\left(D_{1} \times D_{2}\right)$ in the obvious way, we have a closed oriented surface embedded in $\mathbb{R}^{4}$. It is called the closure of $S$ in $\mathbb{R}^{4}$ and denoted by $\widehat{S}$.

When $S$ is described by a motion picture $\left\{b_{t}\right\}_{t \in[0,1]}$, we have a motion picture of the closure $\widehat{S}$ as follows: For each $t \in[0,1]$, assume that $b_{t}$ is lying in the hyperplane $\mathbb{R}^{3} \times\{t\} \subset \mathbb{R}^{3} \times \mathbb{R}^{1}=\mathbb{R}^{4}$ and consider the closure $\widehat{b}_{t}$ of $b_{t}$ in the hyperplane. The trace of the closed braids forms a properly embedded surface in $\mathbb{R}^{3} \times[0,1]$. Since 
$b_{0}$ and $b_{1}$ are trivial $m$-braids, $\widehat{b}_{0}$ and $\widehat{b}_{1}$ are trivial links in $\mathbb{R}^{3} \times\{0\}$ and $\mathbb{R}^{3} \times\{1\}$, respectively. Attaching $m$ trivial disks in the lower side and $m$ trivial disks in the upper side, we have a closed surface in $\mathbb{R}^{4}$, which is the closure of $S$.

Theorem 5 (Viro 29, [12]). Any 2-knot (or closed oriented 2-submanifold of $\mathbb{R}^{4}$ ) is equivalent to the closure of a simple 2-dimensional $\mathrm{m}$-braid for some $\mathrm{m}$.

Definition 6. The braid index of a 2 -knot $K$, denoted by $\operatorname{Braid}(K)$, is the minimum number among the degrees of all simple 2-dimensional braids whose closures are equivalent to $K$.

The following theorem corresponds to the Markov theorem for 1-knots [3, 21].

Theorem 7 (13, 18, 19]). Let $S$ and $S^{\prime}$ be simple 2-dimensional braids. The closure of $S$ is equivalent to that of $S^{\prime}$ if and only if there exists a finite sequence

$$
S=S_{0}, S_{1}, \ldots, S_{n}=S^{\prime}
$$

of simple 2-dimensional braids such that each $S_{i}, i=1,2, \ldots, n$, is obtained from $S_{i-1}$ by a braid ambient isotopy, a conjugation move, a stabilization move or a destabilization move.

Here, two simple 2-dimensional $m$-braids $S$ and $S^{\prime}$ are braid ambient isotopic if they are ambiently isotopic by an ambient isotopy $\left\{h_{t}\right\}_{t \in[0,1]}$ of $D_{1} \times D_{2}$ such that for each $t \in[0,1], h_{t}(S)$ is a (possibly non-simple) 2-dimensional $m$-braid. If $S$ and $S^{\prime}$ are equivalent, then they are braid ambient isotopic. It is unknown whether the converse holds or not. Conjugation, stabilization and destabilization moves are explained in the next section in terms of the chart presentation.

\section{The CHART PRESENTATION}

In this section, we recall the chart presentation of a simple 2-dimensional braid (cf. [8, 10, 19]). An $m$-chart is a finite graph in the interior of a 2-disk $D_{2}$, which may be empty or have hoops (that are closed edges without vertices), satisfying the following conditions:

(i) Every vertex has valency 1, 4, or 6 .

(ii) Every edge is oriented and labeled by an integer from $\{1,2, \ldots, m-1\}$.

(iii) Around each 4-valent vertex, diagonal edges have the same label and are oriented coherently, and the labels $i$ and $j$ of the diagonals satisfy $|i-j|>1$.

(iv) Around each 6-valent vertex, three consecutive edges are oriented inward and the others are oriented outward. These six edges are labeled $i$ and $i+1$ alternately around the vertex for some $i$.

See Figure 2, We do not distinguish two $m$-charts if they are ambiently isotopic in $D_{2}$. A free edge means a single edge whose endpoints are 1-valent vertices.

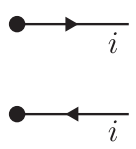

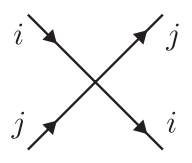

$|i-j|>1$

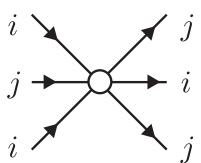

$|i-j|=1$

FIGURE 2. 
Divide $D_{2}$ into two 2-disks $D_{2}^{(1)}$ and $D_{2}^{(2)}$ by a properly embedded arc in $D_{2}$ as in Section 2. For two charts $\Gamma_{1}$ and $\Gamma_{2}$ in $D_{2}$, we denote by $\Gamma_{1} \cdot \Gamma_{2}$ the chart that is the union of a copy of $\Gamma_{1}$ in $D_{2}^{(1)}$ and a copy of $\Gamma_{2}$ in $D_{2}^{(2)}$.

Let $\Gamma$ be an $m$-chart in $D_{2}=I_{1} \times I_{2}$. Put $\ell_{t}=I_{1} \times\{t\}$ for each $t \in I_{2}=[0,1]$. Modifying $\Gamma$ by an isotopy of $D_{2}$, we assume that for all $t$ but a finite number of exceptional values, there are no vertices of $\Gamma$ on $\ell_{t}$ and the intersection of $\ell_{t}$ and $\Gamma$ consists of transverse double points. Also, for each exceptional value $t$, the intersection of $\ell_{t}$ and $\Gamma$ consists of transverse double points and a single point that is a vertex of $\Gamma$ or a maximal or minimal point of an edge of $\Gamma$.

For a regular value $t$, assign each point of the intersection $\ell_{t} \cap \Gamma$ a letter $\sigma_{i}$ (or $\sigma_{i}^{-1}$ ) if its intersecting edge of $\Gamma$ is labeled $i$ and oriented from left to right (or right to left). We read all these letters along $\ell_{t}$ to obtain a word on the standard generators $\sigma_{1}, \ldots, \sigma_{m-1}$ of the $m$-braid group. We denote this braid word by $w_{\Gamma}\left(\ell_{t}\right)$ and call it the intersection braid word along $\ell_{t}$ with respect to the chart $\Gamma$. It is proved in [10] that there is a simple 2-dimensional $m$-braid $S_{\Gamma}$ whose motion picture $\left\{b_{t}\right\}_{t \in[0,1]}$ satisfies the condition that for each regular value $t$, the $m$-braid $b_{t}=S_{\Gamma} \cap(D \times I)_{t}$ is presented by the braid word $w_{\Gamma}\left(\ell_{t}\right)$. It is also proved that the equivalence class of $S_{\Gamma}$ is uniquely determined from (the ambient isotopy class of) the chart $\Gamma$. Conversely, any simple 2-dimensional $m$-braid $S$ is equivalent to $S_{\Gamma}$ for some (not unique) $m$-chart $\Gamma$. In this situation, we say that $\Gamma$ is a chart presentation of $S$, or $S$ is presented by the chart $\Gamma$. Refer to [8, 10, 19] for more details.

Example 8. The top of Figure 3 shows an example of a 4-chart, and the 2dimensional braid presented by this chart is depicted in the middle and bottom of the figure by the motion picture, where $t_{1}, \ldots, t_{4}$ are exceptional values.

Local operations on $m$-charts listed below are called CI-, CII-, and CIII-moves, respectively. A C-move is one of them or its inverse. Two $m$-charts are $\mathrm{C}$-move equivalent if they are related by a sequence of C-moves (up to ambient isotopy in $\left.D_{2}\right)$. Let $\Gamma$ be an $m$-chart in $D_{2}$.

(CI) For a 2-disk $E$ in $D_{2}$ such that $\Gamma \cap E$ has no 1-valent vertices, change $\Gamma \cap E$ arbitrarily as long as it has no 1 -valent vertices.

(CII) Suppose that an edge $e$ connects a 4 -valent vertex $v^{4}$ and a 1 -valent vertex $v^{1}$. Remove $e$ and $v^{4}$, attach $v^{1}$ to the edge of $v^{4}$ opposite to $e$, and connect the other edges in a natural way (see Figure 4).

(CIII) Let a 1 -valent vertex $v^{1}$ and a 6 -valent vertex $v^{6}$ be connected by an edge $e$. Suppose that $e$ is not the middle edge of a set of three consecutive edges attaching to $v^{6}$ which are oriented in the same direction. Then, remove $e$ and $v^{6}$, attach $v^{1}$ to the edge of $v^{6}$ opposite to $e$, and connect the other edges in a natural way (see Figure 4).

Figure 5 shows two examples of CI-moves. The first one in the figure is called a channel change move.

Theorem 9 (10, 15). Two $m$-charts $\Gamma$ and $\Gamma^{\prime}$ are $C$-move equivalent if and only if the simple 2-dimensional $m$-braids presented by $\Gamma$ and $\Gamma^{\prime}$ are equivalent.

We consider two more operations on charts. Let $\Gamma$ be an $m$-chart in $D_{2}$.

(MI) Add some hoops (simple loops) parallel to $\partial D_{2}$ surrounding $\Gamma$. Each of the hoops is oriented and labeled by an integer from $\{1, \ldots, m-1\}$ arbitrarily. 

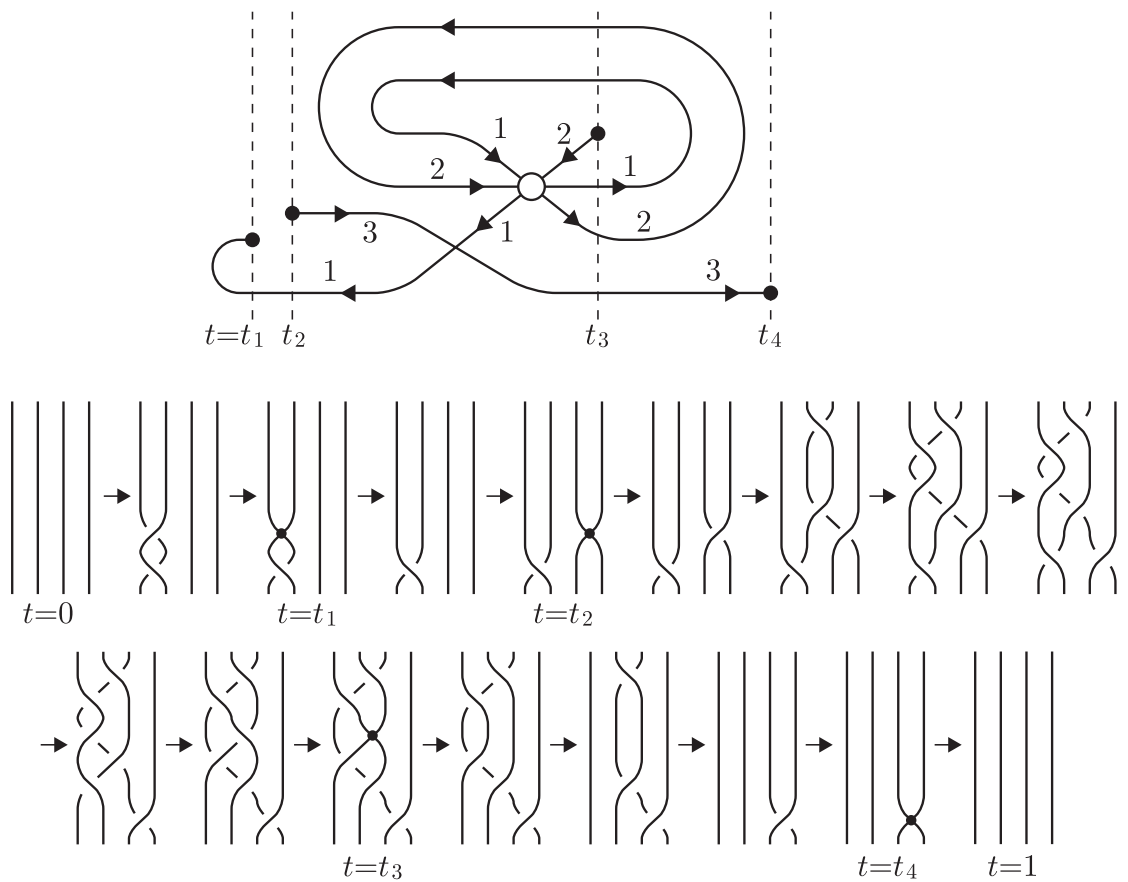

FiguRE 3.

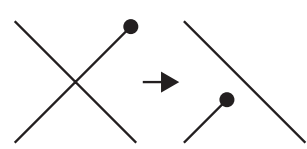

(CII)

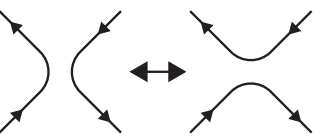

channel change move
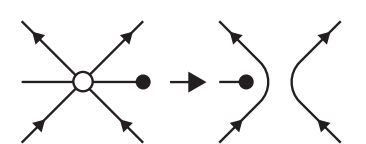

(CIII)

Figure 4.
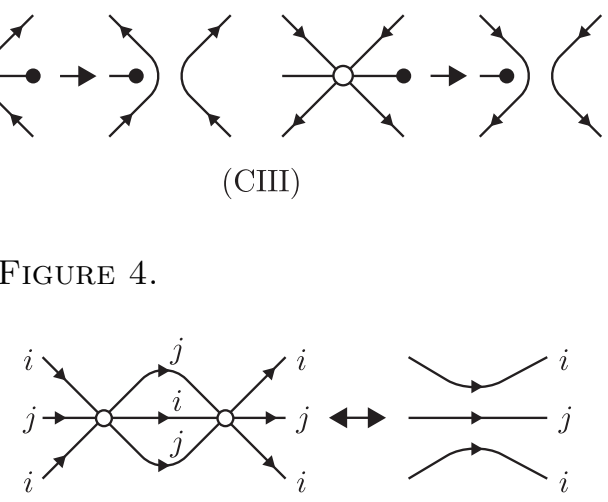

$|i-j|=1$

FiguRE 5 .

(MII) Insert a free edge labeled $m$ near $\partial D_{2}$, and regard the union of $\Gamma$ and the free edge as an $(m+1)$-chart.

Let $\Gamma$ be a chart presentation of a simple 2 -dimensional $m$-braid $S$. We say that a simple 2-dimensional $m$-braid $S^{\prime}$ is obtained from $S$ by a conjugation move if $S^{\prime}$ has a chart presentation that is obtained from $\Gamma$ by an MI-move or its inverse. A simple 2-dimensional $(m+1)$-braid $S^{\prime}$ is obtained from $S$ by a stabilization move if $S^{\prime}$ has a chart presentation that is obtained from $\Gamma$ by an MII-move. A destabilization move is the inverse operation of a stabilization move. See Figures 6 and 7 for 
the motion pictures. These definitions stated in terms of the chart presentation 17] are compatible with the original definitions in [13] stated geometrically, and the definitions in [18] stated in terms of braid systems. Refer to [19] for details and for a complete proof of Theorem 7. Therefore, if two charts are related by a sequence of C-moves, MI-moves and MII-moves, then the closures of their presenting 2-dimensional braids are equivalent.

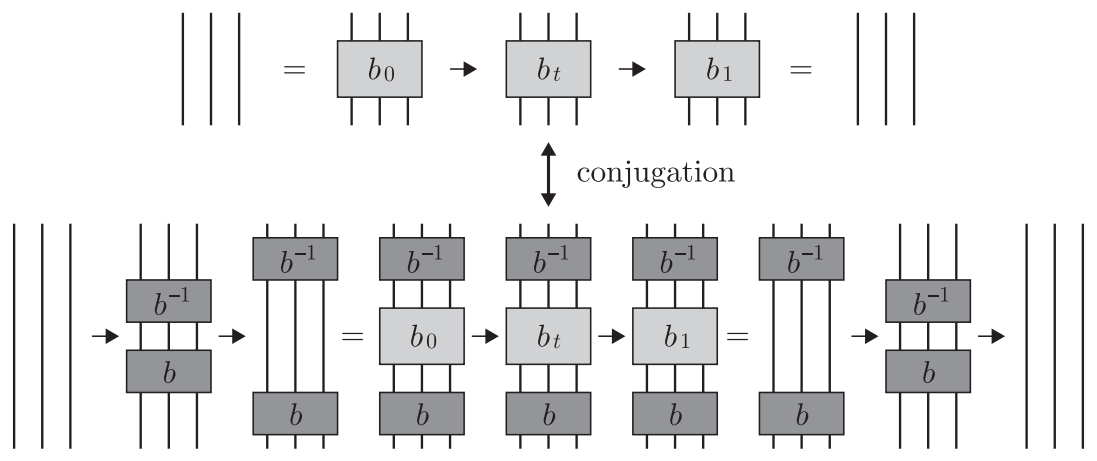

FiguRE 6.

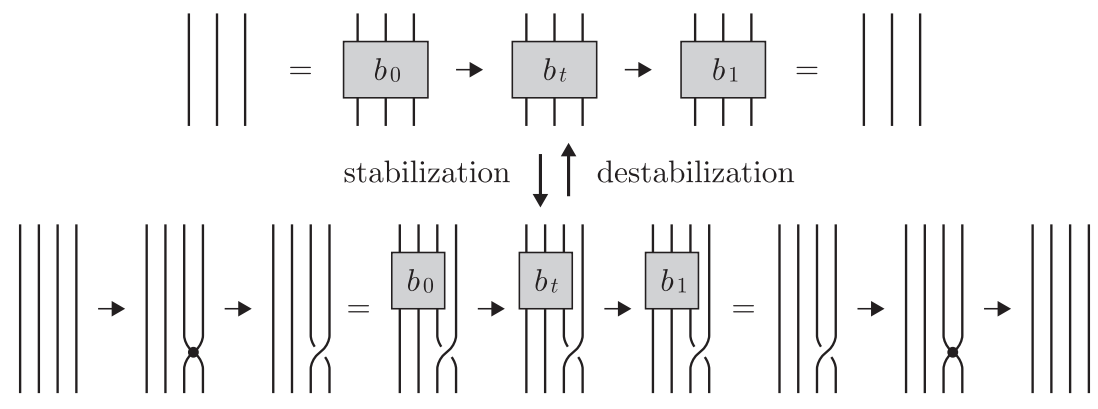

FiguRE 7.

\section{The Composite of 2-Dimensional BRAids}

Let $p$ and $q$ be non-negative integers. For a (1-dimensional) $m$-braid $b$, we denote by $\iota_{p}^{q}(b)$ the $(p+m+q)$-braid that is obtained from $b$ by introducing $p$ trivial strands on the left side of $b$ and $q$ trivial strands on the right side. In other words, when a braid $b$ is expressed by a word on the standard generators $\sigma_{1}, \ldots, \sigma_{m-1}$, the $(p+m+q)$-braid $\iota_{p}^{q}(b)$ is obtained by shifting the subscripts $i$ of $\sigma_{i}$ to $i+p(i=1, \ldots, m-1)$, and considering these to be generators in the braid group $B_{p+m+q}$.

The composite or the connected sum of an $m_{1}$-braid $b_{1}$ and an $m_{2}$-braid $b_{2}$, denoted by $b_{1} \# b_{2}$, is the $\left(m_{1}+m_{2}-1\right)$-braid

$$
\iota_{0}^{m_{2}-1}\left(b_{1}\right) \cdot \iota_{m_{1}-1}^{0}\left(b_{2}\right)
$$

(see Figure 8). The closure of $b_{1} \# b_{2}$ is a connected sum of the closures of $b_{1}$ and $b_{2}$. The dotted circle in the figure indicates a decomposing 2 -sphere in $\mathbb{R}^{3}$ for the 
connected sum; namely, it shows the equatorial cross-section of the decomposing 2 -sphere with respect to the plane in $\mathbb{R}^{3}$ on which the knot diagram is constructed.
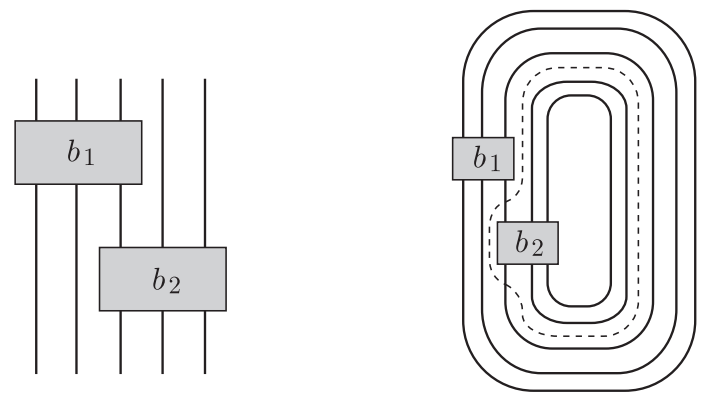

FiguRE 8.

The notion of the composite of 1-dimensional braids is generalized to that of 2dimensional braids as follows: For a 2 -dimensional $m$-braid $S$, we denote by $\iota_{p}^{q}(S)$ the 2-dimensional $(p+m+q)$-braid that is obtained from $S$ by introducing $p$ trivial 2-disks on the left side of $S$ and $q$ trivial 2-disks on the right side. When $S$ is described by a motion picture $\left\{b_{t}\right\}_{t \in[0,1]}$, then the 2-dimensional $(p+m+q)$-braid $\iota_{p}^{q}(S)$ is described by the motion picture $\left\{\iota_{p}^{q}\left(b_{t}\right)\right\}_{t \in[0,1]}$. Note that we use the same notation $\iota_{p}^{q}$ for 1- and 2-dimensional braids, but it will cause no confusion.

The composite or the connected sum of a 2-dimensional $m_{1}$-braid $S_{1}$ and a 2 dimensional $m_{2}$-braid $S_{2}$, denoted by $S_{1} \# S_{2}$, is the 2 -dimensional $\left(m_{1}+m_{2}-1\right)$ braid

$$
\iota_{0}^{m_{2}-1}\left(S_{1}\right) \cdot \iota_{m_{1}-1}^{0}\left(S_{2}\right) .
$$

In other words, a motion picture of the composite $S_{1} \# S_{2}$ is obtained from the motion pictures $\left\{b_{t}^{(1)}\right\}_{t \in[0,1]}$ and $\left\{b_{t}^{(2)}\right\}_{t \in[0,1]}$ of $S_{1}$ and $S_{2}$ by taking the composite braid $b_{t}^{(1)} \# b_{t}^{(2)}$ for each $t \in[0,1]$.

Lemma 10 ([17]). The closure of the composite $S_{1} \# S_{2}$ is a connected sum of the closures of $S_{1}$ and $S_{2}$.

Proof. The trace of the decomposing 2-spheres for the closures of $b_{t}^{(1)} \# b_{t}^{(2)}$ in $\mathbb{R}^{3} \times$ $\{t\} \subset \mathbb{R}^{4}$ for all $t \in[0,1]$ (as in Figure 8) forms an embedded 3-manifold in $\mathbb{R}^{3} \times$ $[0,1] \subset \mathbb{R}^{4}$ which is homeomorphic to $S^{2} \times[0,1]$. Attaching a 3 -ball in the upper side and a 3 -ball in the lower side in an obvious way, we have a decomposing 3 -sphere in $\mathbb{R}^{4}$ for a connected sum of the closures of $S_{1}$ and $S_{2}$.

Lemma 2 follows from this lemma immediately.

In terms of the chart presentation, the composite of 2-dimensional braids is stated as follows: For an $m$-chart $\Gamma$ and non-negative integers $p$ and $q$, let $\iota_{p}^{q}(\Gamma)$ be the $(p+m+q)$-chart obtained from $\Gamma$ by adding $p$ to the label of every edge of $\Gamma$ and regarding the chart as an $(p+m+q)$-chart. This notion, $\iota_{p}^{q}(\Gamma)$, is compatible with the notion of $\iota_{p}^{q}(S)$ for a 2-dimensional braid $S$ : If a 2-dimensional $m$-braid $S$ has a chart presentation $\Gamma$, then the chart $\iota_{p}^{q}(\Gamma)$ is a chart presentation of the 2-dimensional $(p+m+q)$-braid $\iota_{p}^{q}(S)$. For an $m_{1}$-chart $\Gamma_{1}$ and an $m_{2}$-chart $\Gamma_{2}$, we denote by $\Gamma_{1} \# \Gamma_{2}$ the $\left(m_{1}+m_{2}-1\right)$-chart

$$
\iota_{0}^{m_{2}-1}\left(\Gamma_{1}\right) \cdot \iota_{m_{1}-1}^{0}\left(\Gamma_{2}\right) .
$$


Then $\Gamma_{1} \# \Gamma_{2}$ is a chart presentation of $S_{1} \# S_{2}$, where $S_{1}$ and $S_{2}$ are 2-dimensional braids presented by $\Gamma_{1}$ and $\Gamma_{2}$.

The following lemma is equivalent to Lemma 10

Lemma 11 ([17). Let $K_{i}$ be a closed oriented surface in $\mathbb{R}^{4}$ presented by an $m_{i}$ chart $\Gamma_{i}(i=1,2)$. Then the $\left(m_{1}+m_{2}-1\right)$-chart $\Gamma_{1} \# \Gamma_{2}$ presents a connected sum $K_{1} \# K_{2}$.

\section{Proof of Theorem 3}

Let $v$ be a 1 -valent vertex of a chart $\Gamma$ in $D_{2}$. The label of $v$ stands for the label of the edge adjacent to $v$. The sign of $v$ is +1 (or -1 ) if the adjacent edge is oriented outward from $v$ (or toward $v$ ). We say that $v$ is outermost if we can connect the vertex $v$ and a point of $\partial D_{2}$ by a simple arc $\alpha$ such that $\alpha \cap \Gamma=\{v\}$.

Lemma 12. Let $\Gamma$ be an $m$-chart with 1-valent vertices, where $m \geq 2$. For any $\varepsilon \in\{+1,-1\}$ and $k \in\{1, \ldots, m-1\}$, one can transform $\Gamma$ by $C$-moves and $M I$ moves such that there exists an outermost 1 -valent vertex with sign $\varepsilon$ and label $k$.

Proof. Note that half of the 1-valent vertices have positive signs and the other half have negative signs; cf. [10]. Choose a 1 -valent vertex whose sign is $\varepsilon$, say $v$. The composition of a CI-move and a CIII-move illustrated in Figure 9 changes the label $i$ of $v$ into an integer $j$ with $|i-j|=1$ and $1 \leq j \leq m-1$. Applying this inductively, we may assume that the label of $v$ is the given integer $k$. Using an MI-move and a CI-move, we can make the vertex $v$ to be outermost as follows: Consider a simple arc $\alpha$ from $v$ to a point of $\partial D_{2}$ intersecting $\Gamma$ transversely, and read the intersection braid word $w_{\Gamma}(\alpha)$ along $\alpha$ (ignoring the starting point $v$ ) as in Section 3. Add some hoops parallel to $\partial D_{2}$ oriented and labeled such that the intersection braid word of these hoops along $\alpha$ is the inverse of $w_{\Gamma}(\alpha)$. This is an MI-move. Applying channel change moves along $\alpha$ as in Figure 10, which is a CI-move, we can remove the intersection of the chart and the arc $\alpha$, except for the vertex $v$.

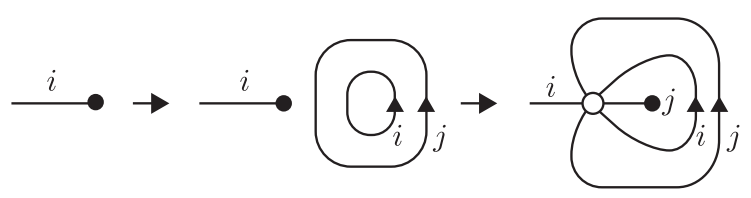

FIGURE 9.

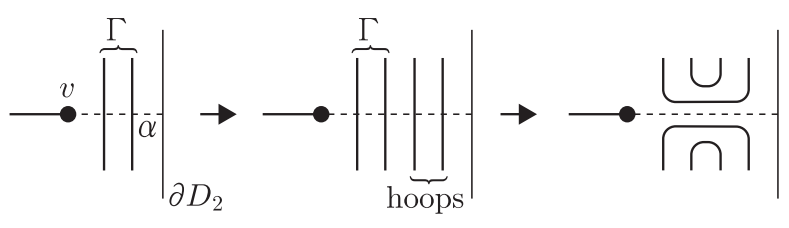

Figure 10.

Let $\Gamma$ and $E$ be an $m$-chart and a 2-disk in $D_{2}$ respectively such that $\Gamma \cap \partial E$ is empty or consists of transverse double points missing the vertices of $\Gamma$. Then we call the intersection $\Gamma \cap E$ a subchart of $\Gamma$. 
Lemma 13. Let $m$ and $\ell$ be integers with $1<m<\ell$. Let $\Gamma$ be an $\ell$-chart which has a subchart $\Lambda$ whose edges are labeled by integers in $\{m, m+1, \ldots, \ell-1\}$ as shown in the left of Figure 11. Let $\Gamma^{\prime}$ be the $\ell$-chart obtained from $\Gamma$ by the replacement illustrated in Figure 11, where $\Lambda^{\prime}$ is the subchart obtained from $\Lambda$ by shifting all of the labels by -1 . Then $\Gamma$ and $\Gamma^{\prime}$ present the same 2-dimensional braid.

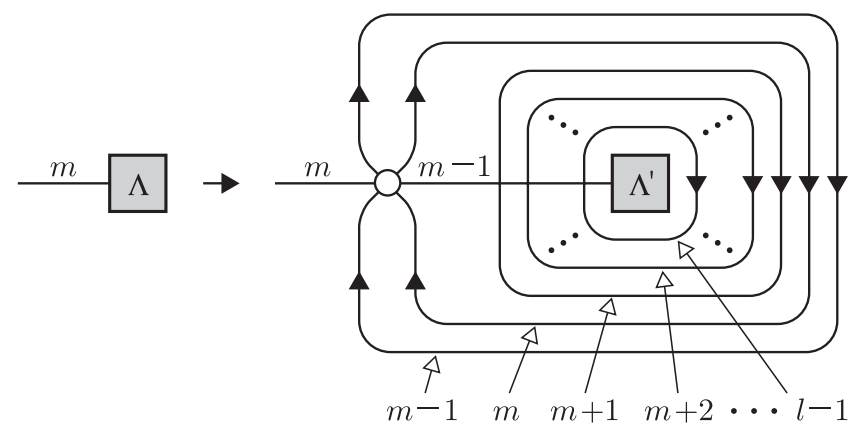

FiguRE 11.

Lemma 14. Let $m$ and $\ell$ be integers with $1<m<\ell$. Let $\Gamma$ be an $\ell$-chart which has a subchart as shown on the left side of Figure 12, and let $\Gamma^{\prime}$ be the $\ell$-chart obtained from $\Gamma$ by the replacement illustrated in Figure 12. Then $\Gamma$ and $\Gamma^{\prime}$ present the same 2-dimensional braid.

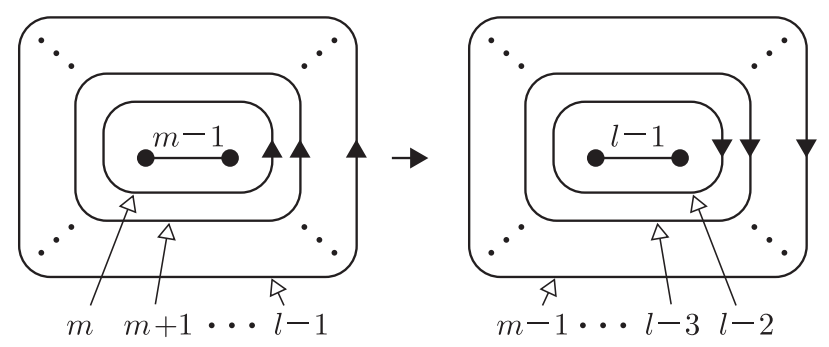

FiguRE 12.

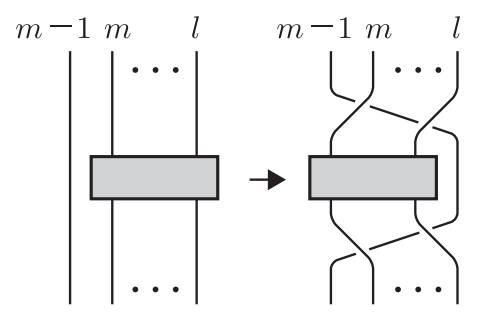

(i)

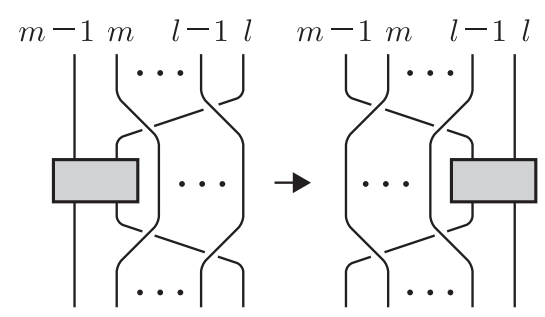

(ii)

FiguRE 13. 

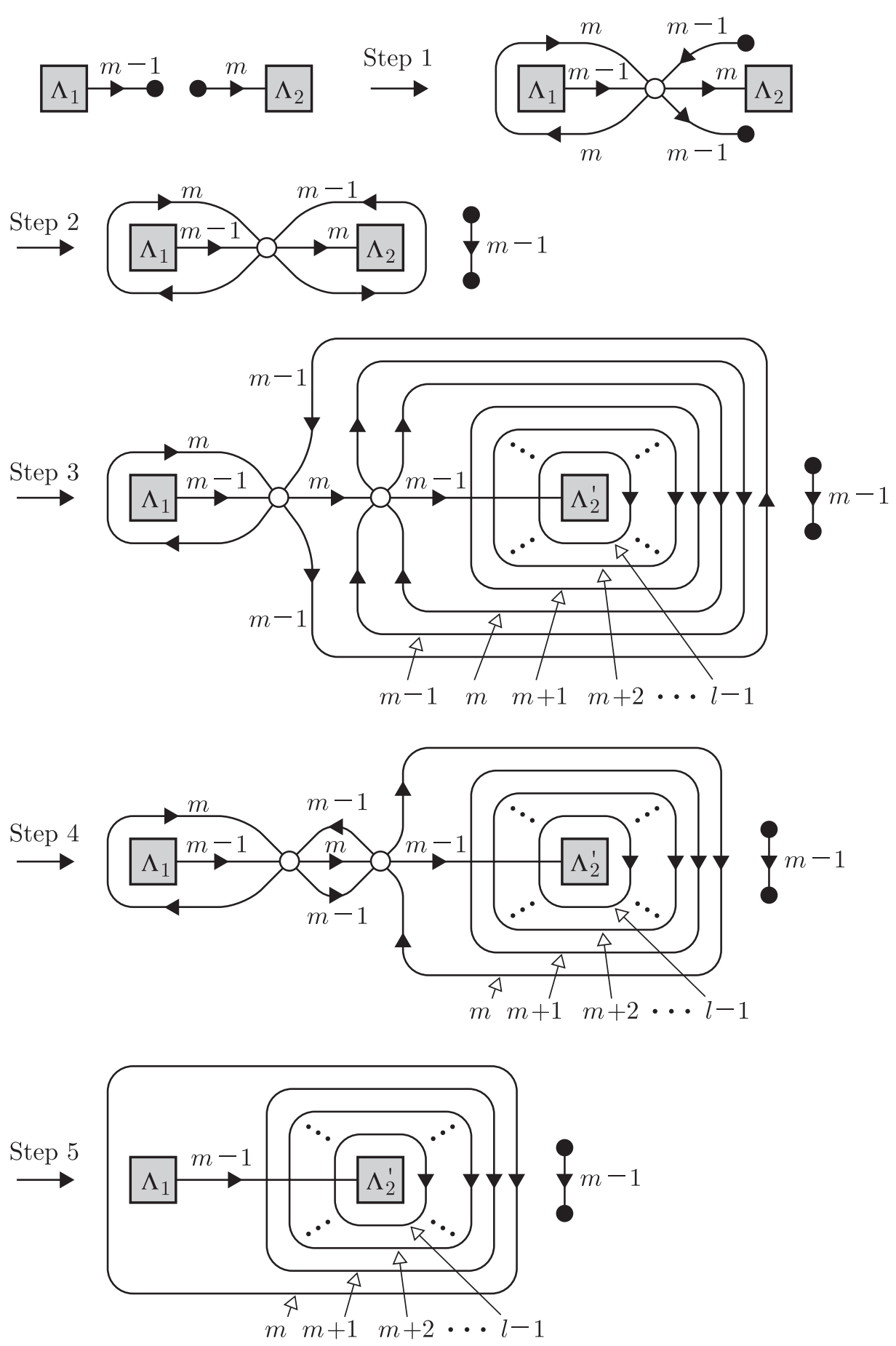

Figure 14. 

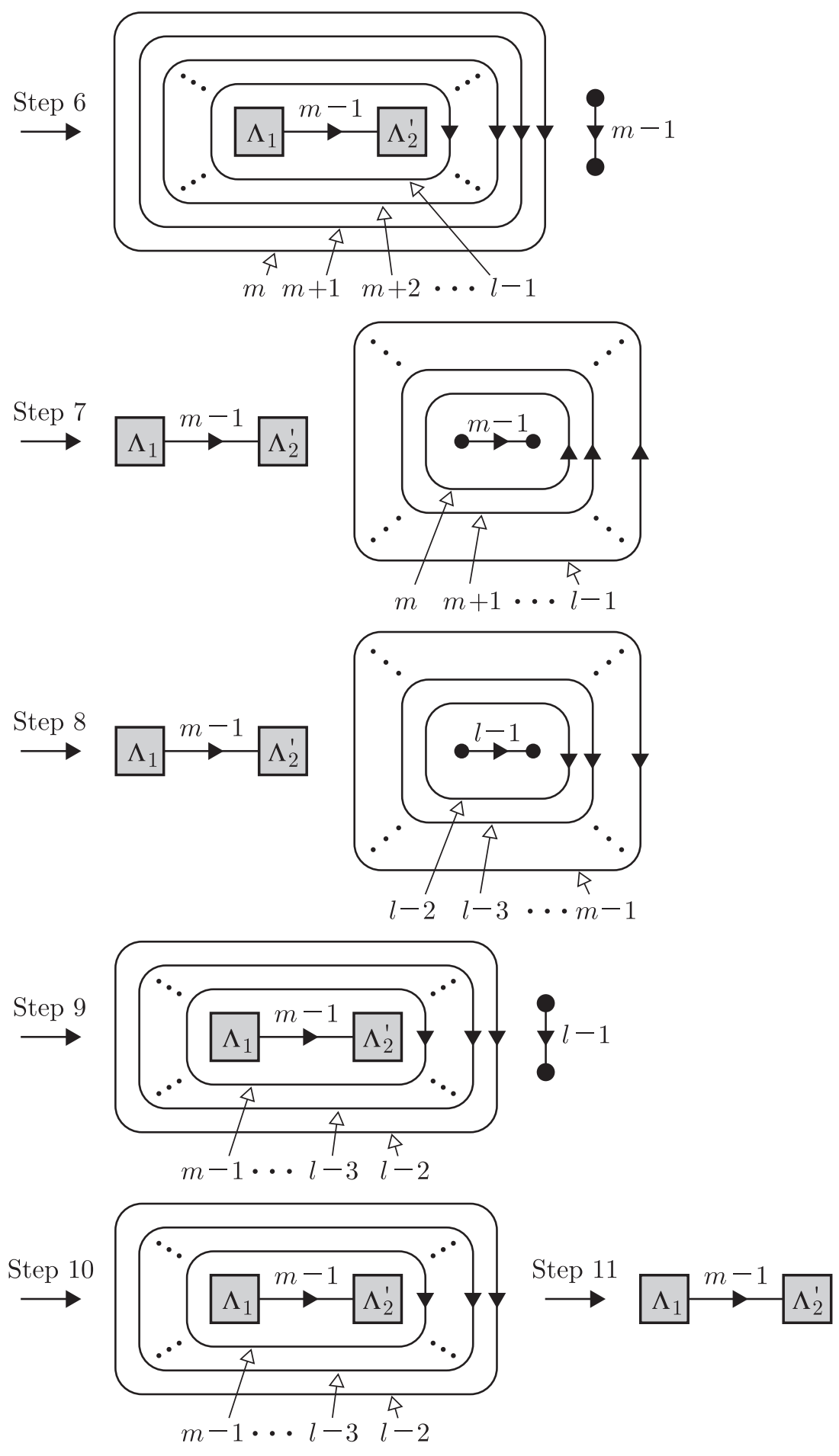

Figure 15. 
Proofs of Lemmas 13 and 14. Since the braid monodromies of $\Gamma$ and $\Gamma^{\prime}$ are the same, they present the same (up to equivalence) 2-dimensional braid (see [14, 15, 19] for the definition of the braid monodromy and a proof of the fact that the braid monodromy determines the 2-dimensional braid).

The ideas of Lemmas 13 and 14 are schematized as in Figure 13(i) and (ii), respectively.

Proof of Theorem 3, Let $K_{i}$ be a non-trivial 2-knot and $\Gamma_{i}$ an $m_{i}$-chart presenting $K_{i}(i=1,2)$. It is sufficient to prove that the connected sum $K_{1} \# K_{2}$ has a chart presentation by an $\left(m_{1}+m_{2}-2\right)$-chart. Note that both of $\Gamma_{1}$ and $\Gamma_{2}$ have 1-valent vertices. (If $\Gamma_{i}$ has no 1 -valent vertices, then the 2 -dimensional braid presented by $\Gamma_{i}$ is trivial [10, and $K_{i}$ is the union of $m_{i}$ trivial 2-spheres in $\mathbb{R}^{4}$. Since $K_{i}$ is a non-trivial 2-knot, this case does not occur.) Using Lemma 12 we may assume that the $m_{1}$-chart $\Gamma_{1}$ has an outermost 1 -valent vertex with sign -1 and label $m_{1}-1$ and that the $m_{2}$-chart $\Gamma_{2}$ has an outermost 1-valent vertex with sign +1 and label 1 . Let $\Gamma$ be the composite chart $\Gamma_{1} \# \Gamma_{2}=\iota_{0}^{m_{2}-1}\left(\Gamma_{1}\right) \cdot \iota_{m_{1}-1}^{0}\left(\Gamma_{2}\right)$. This is an $\left(m_{1}+m_{2}-1\right)$-chart presenting the connected sum $K_{1} \# K_{2}$. Put $m=m_{1}$ and $\ell=m_{1}+m_{2}-1$. The chart $\Gamma$ is shown as in the top left of Figure 14, where $\Lambda_{1}$ is a subchart whose edges are labeled by integers in $\{1, \ldots, m-1\}$ and $\Lambda_{2}$ is a subchart whose edges are labeled by integers in $\{m, m+1, \ldots, \ell-1\}$. Transform the chart $\Gamma$ as in Figures 14 and 15. (Step 1) It is a CIII-move; (Step 2) It is a channel change move which is a CI-move; (Step 3) Do the replacement established in Lemma 13. (Step 4) It is a channel change move; (Step 5) It is a CI-move as in Figure 5: (Step 6) It is a combination of C-moves; (Step 7) It is an MI-move with channel change moves; (Step 8) It is the replacement established in Lemma 14, (Step 9) It is an MI-move with channel change moves. Note that after this step, the chart has a free edge of label $\ell-1$ and the other edges of the chart are labeled by integers smaller than $\ell-1$. (Step 10) Apply the inverse operation of an MII-move and obtain the $(\ell-1)$-chart in the figure. (Step 11) It is an MI-move. Thus the $(\ell-1)$-chart illustrated in the right bottom of Figure 15 is a chart presentation of $K_{1} \# K_{2}$.

\section{EXAMPLES}

Let $n$ be an odd integer with $n \geq 3$ and let $\Gamma_{n}$ be the 3 -chart illustrated in Figure 16 which consists of two free edges with label 1 and $n$ parallel hoops with label 2 oriented in the same direction surrounding one of the free edges. We denote by $K_{n}$ the 2 -knot presented by the chart $\Gamma_{n}$ (which was denoted by $S_{n, 1}$ in [16]). Note that $K_{n}$ is the 2-knot obtained from a $(2, n)$-torus knot by Artin's spinning (cf. [8, 10, 16, 19]).

Lemma 15. Braid $\left(K_{n}\right)=3$ for any odd integer $n \geq 3$. If $n \neq n^{\prime}$, then $K_{n}$ is not equivalent to $K_{n^{\prime}}$. Hence, there exist an infinite series of 2-knots of braid index 3 .

Proof. The first Alexander module, the $\Lambda=\mathbb{Z}\left[t, t^{-1}\right]$-module of the homology $H_{1}(\widetilde{E} ; \mathbb{Z})$ of the universal abelian covering $\widetilde{E} \rightarrow E$ of the knot exterior $E$ of the 2 -knot $K_{n}$ is isomorphic to that of a $(2, n)$-torus knot. Thus, we see that $K_{n}$ is non-trivial and that $K_{n}$ is not equivalent to $K_{n^{\prime}}$ if $n \neq n^{\prime}$. Since any non-trivial 2-knot has braid index greater than 2, we have the result.

Lemma 16. $\operatorname{Braid}\left(K_{n} \# K_{n}\right)=4$ for any odd integer $n \geq 3$. 


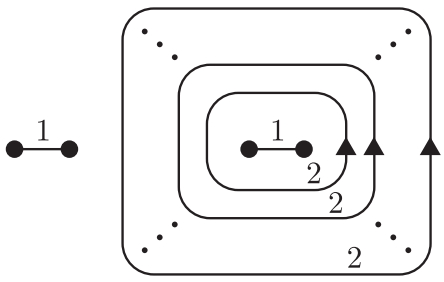

FIGURE 16.

Proof. By Theorem 3 and Lemma [15, we have $\operatorname{Braid}\left(K_{n} \# K_{n}\right) \leq 4$. Using the standard form of a 3-braid 2-knot [16, it is seen that any 3-braid 2-knot is a 1fusion ribbon 2-knot, that is, it is obtained from a pair of trivial 2-spheres in $\mathbb{R}^{4}$ by surgery along a single 1-handle. So its first Alexander module must be cyclic. On the other hand, the first Alexander module of $K_{n} \# K_{n}$ is the direct sum of two copies of $\Lambda /\left(f_{n}\right)$, which is not cyclic, where $f_{n}$ is the Alexander polynomial of a $(2, n)$-torus knot. Hence, we have $\operatorname{Braid}\left(K_{n} \# K_{n}\right)>3$.

Proof of Corollary 4. Consider the family $\left\{K_{n} \# K_{n}\right\}$ for odd integers $n \geq 3$. If $n \neq n^{\prime}$, then $K_{n} \# K_{n}$ and $K_{n^{\prime}} \# K_{n^{\prime}}$ are not equivalent, since their first Alexander modules are not isomorphic. In particular, $K_{3} \# K_{3}$ is the 2 -knot obtained from a granny knot by Artin's spinning.

Questions. Do there exist 2-knots $K_{1}$ and $K_{2}$ with

$$
\operatorname{Braid}\left(K \# K^{\prime}\right)<\operatorname{Braid}\left(K_{1}\right)+\operatorname{Braid}\left(K_{2}\right)-2 ?
$$

In particular, do there exist 2-knots $K_{1}$ and $K_{2}$ with

$$
\operatorname{Braid}\left(K_{1} \# K_{2}\right)=\operatorname{Braid}\left(K_{1}\right)=\operatorname{Braid}\left(K_{2}\right)=3 ?
$$

\section{REFERENCES}

[1] J. W. Alexander, A lemma on systems of knotted curves, Proc. Nat. Acad. Sci. USA 9 (1923), 93-95.

[2] E. Artin, Zur Isotopie Zwei-dimensionaler Flaächen im $R_{4}$, Abh. Math. Sem. Univ. Hamburg 4 (1925), 174-177.

[3] J. S. Birman, "Braids, Links, and Mapping Class Groups", Ann. Math. Studies 82, 1974, Princeton Univ. Press, Princeton, N.J. MR0375281 (51:11477)

[4] J. S. Birman and W. Menasco, Studying links via closed braids IV: Composite links and split links, Invent. Math. 102 (1990), 115-139. MR1069243 (92g:57010a)

[5] J.S. Carter and M. Saito, Knotted surfaces, braid moves and beyond, in "Knots and Quantum Gravity", 191-229, Oxford Science Publishing, 1994. MR1309921 (95m:57036)

[6] Knot diagrams and braid theories in dimension 4, in "Real and Complex Singularities" Papers from 3rd International Workshop (São Carlos 1994), 112-147, Pitman Res. Notes Math. Ser. 333, 1995. MR1478515 (98g:57037)

[7] , Braids and movies, J. Knot Theory Ramifications 5 (1996), 589-608. MR1414089 $(97 \mathrm{j}: 57028)$

[8] "Knotted Surfaces and Their Diagrams", Math. Surveys and Monographs, Amer. Math. Soc. vol. 55, 1998. MR1487374 (98m:57027)

[9] F. González-Acuña, A characterization of 2-knot groups, Rev. Mat. Iberoamericana 10 (1994), 221-228. MR.1286475 (95f:57042)

[10] S. Kamada, Surfaces in $R^{4}$ of braid index three are ribbon, J. Knot Theory Ramifications 1 (1992), 137-160. MR1164113 (93h:57039) 
[11] _ 2-dimensional braids and chart descriptions, in "Topics in Knot Theory", 277287, NATO ASI Series C 399 (Erzurum/Turkey 1992), Kluwer Academic Publisher, 1992. MR.1257915

[12] - A characterization of groups of closed orientable surfaces in 4-space, Topology 33 (1994), 113-122. MR1259518(95a:57002)

[13] _ Alexander's and Markov's theorems in dimension four, Bull. Amer. Math. Soc. 31 (1994), 64-67. MR.1254074 (94j:57023)

[14] $\_$, On braid monodromies of non-simple braided surfaces, Math. Proc. Camb. Phil. Soc. 120 (1996), 237-245. MR.1384466 (97f:57002)

[15] - An observation of surface braids via chart description, J. Knot Theory Ramifications, 4 (1996), 517-529. MR.1406718 (97j:57009)

[16] Standard forms of 3-braid 2-knots and their Alexander polynomials, Michigan Math. J. 45 (1998), 189-205. MR1617423 (99h:57048)

[17] _ Unknotting immersed surface-links and singular 2-dimensional braids by 1-handle surgeries, Osaka J. Math. 36 (1999), 33-49. MR1670738 (2000e:57040)

[18] _ Arrangement of Markov moves for 2-dimensional braids, in "Low Dimensional Topology" (Madeira, Portugal, 1998), Contemp. Math., 233, Amer. Math. Soc., 1999, 197213. MR 1701684 (2000e:57039)

[19] _ "Braid and Knot Theory in Dimension Four", Math. Surveys and Monographs, Amer. Math. Soc., 2002. MR1900979(2003d:57050)

[20] A. Kawauchi, "A Survey of Knot Theory", Birkhäuser Verlag, Basel·Boston·Berlin, 1996. MR.1417494 (97k:57011)

[21] A. A. Markov, Uber die freie Aquivalenz der geschlossner Zopfe, Rec. Soc. Math. Moscou 1 (1935), 73-78.

[22] B. Moishezon, Stable branch curves and braid monodromies, in "Algebraic Geometry", Lect. Notes in Math. 862, 1981, 107-192. MR0644819 (83c:14008)

[23] B. Moishezon and M. Teicher, Braid group technique in complex geometry I, line arrangements in $\mathbf{C} P^{2}$, Contemp. Math. 78 (1988), 425-555 MR0975093 (90f:32014)

[24] F. Hosokawa and A. Kawauchi, Proposals for unknotted surfaces in four-spaces, Osaka J. Math. 16 (1979), 233-248. MR0527028 (81c:57018)

[25] L. Rudolph, Braided surfaces and Seifert ribbons for closed braids, Comment. Math. Helv. 58 (1983), 1-37. MR0699004 (84j:57006)

[26] Some topologically locally-flat surfaces in the complex projective plane, Comment. Math. Helv. 59 (1984), 592-599. MR0780078 (86h:57019)

[27] Special positions for surfaces bounded by closed braids Rev. Mat. Iberoamericana 1 (1985), 93-133. MR0836285 (88a:57018)

[28] D. Roseman, The spun square knot is the spun granny knot, Bol. Soc. Mat. Mexicana (2) 20 (1975), 49-55. MR0515725 (58:24282)

[29] O. Ya. Viro, Lecture given at Osaka City University, September, 1990.

[30] E. C. Zeeman, Twisting spun knots, Trans. Amer. Math. Soc. 115 (1965), 471-495. MR0195085 (33:3290)

Department of Mathematics, Hiroshima University, Higashi-Hiroshima, 739-8526, JAPAN

E-mail address: kamada@math.sci.hiroshima-u.ac.jp

Department of Mathematics, Chiba University, Inage, Chiba, 263-8522, Japan

E-mail address: satoh@math.s.chiba-u.ac.jp

Japan Tokushima Prefectural, Mental Health \& Welfare Center, 3-80 Shinkura, TOKUSHIMA, 770-0855, JAPAN

E-mail address: manabu12@khaki.plala.or.jp 